\author{
L.A. BULAVIN,${ }^{1,2}$ D.A. GAVRYUSHENKO,${ }^{1}$ K.V. TARADII,${ }^{1}$ N.A. ATAMAS ${ }^{1}$, \\ V.M. SYSOEV ${ }^{1}$ \\ 1 Taras Shevchenko National University of Kyiv \\ (2, Prosp. Academician Glushkov, Kyiv 03022, Ukraine) \\ ${ }^{2}$ Institute for Safety Problems of Nuclear Power Plants, Nat. Acad. of Sci. of Ukraine \\ (12, Lysogirska Str., Bld. 106, Kyiv 03028, Ukraine)
}

\title{
INFLUENCE OF RADIATION ON THE PHASE TRANSITION TEMPERATURE IN LIQUIDS
}

\begin{abstract}
The influence of radiation on the thermodynamic properties of liquid systems that are governed by the radiation-induced change in the chemical potentials of the liquid and its components has been studied. The irradiation of coexisting phases in the stationary state is shown to result in a shift of the phase transition point parameters. The temperature shift of the first-order phase transition under the influence of radiation is evaluated with regard for both the entropy and interaction factors in the chemical potential of the system.
\end{abstract}

Ke ywords: radiation, phase transition, multicomponent system.

\section{Introduction}

In the last decades, a significant number of researches dealing with the influence of radiation on physical systems in various aggregate states were carried out [1-4]. In the majority of corresponding works, their authors considered the formation of defects in the crystalline state of a substance [5-7]. At the same time, the number of works dealing with changes in the structure and the thermodynamic parameters of a medium in the liquid state is few [8]. Recently, there appeared not only researches, where the formation of radiation-induced defects at the "solid-liquid" interface was considered [9], but also researches of the liquid-vapor phase transition [10]. In addition, the substantial attention was given to a change of the phase transition temperature in liquids with a confined geometry (see, e.g., work [11]) and to the changes in the phase transition evolution in medicobiological systems $[12,13]$.

However, the study of modifications in the structure and the thermodynamic parameters of liquid systems, as well as the phase equilibrium parameters, under the influence of radiation is a very challenging problem. In particular, the creation of nuclear reactors of the fourth generation [14] requires the further development of radiation physics of liquids and ra-

(C) L.A. BULAVIN, D.A. GAVRYUSHENKO,

K.V. TARADII, N.A. ATAMAS', V.M. SYSOEV, 2016

ISSN 2071-0194. Ukr. J. Phys. 2016. Vol. 61, No. 9 diation materials science. A special place among nuclear reactors of the new generation is occupied by molten salt reactors (MSRs). Their fuel is a radioactive melt, namely, chemical compounds of a radioactive element (uranium or plutonium) with fluorine, which are dissolved in a melt of inorganic salts $(\mathrm{Li}$, $\mathrm{Na}, \mathrm{KF}$, and others) [15]. The interest to MSRs is associated, first of all, with other physical processes that occur in those installations. As a consequence, their exploitation requires that qualitatively different constructional materials should be applied. The melts of fluoride salts that circulate through the active zone of salt reactors are exposed to radiation fluxes of neutrons, electrons, $\gamma$-quanta, and decay debris. The interaction of radiation with atoms in the melt stimulates a considerable number of various processes, which gives rise to changes in the physical and physico-chemical properties of the melt and a modification of the interaction of radiation with the materials, of which the solid structural elements of a nuclear power installation are made. As a result of those processes, the parameters of constructional materials and the conditions of their exploitation can change as well [16].

Various biological systems are also often subjected to irradiation. The influence of ionizing radiation on live organisms, tissues, cells, etc. has already been studied for a long period. At the same time, not enough attention was paid in those researches to the 
influence of radiation on the liquids that are contained in biological objects, despite that this influence can be substantial. The purpose of this work is the study of the influence of radiation on those thermodynamic properties of liquids and liquid systems that are governed by changes in the chemical potentials of the liquid system and its components under the action of radiation.

\section{Radiation-Induced Change of the First-Order Phase Transition Temperature}

Irradiation of a liquid medium gives rise to an appreciable change in its equilibrium thermodynamic properties. The thermodynamic properties of the medium are known to be connected with its structural properties. Owing to irradiation, the thermodynamic equilibrium in the liquid becomes violated, and the liquid structure undergoes a reorganization. The restoration of the equilibrium structure in a liquid is accompanied by various relaxation processes; mainly, this is the structural relaxation. The equilibrium and nonequilibrium properties of the liquid substantially depend on the character of these relaxation processes. However, the issue concerning a detailed analysis of the relaxation processes and the determination of their contribution to the dynamical values of thermodynamic parameters remains open [17].

The state of a liquid system under irradiation is not the equilibrium one, because radiation induces a permanent deviation from the equilibrium. In the framework of the approach proposed in work [18], let us consider the case where a biphasic system (e.g., liquid and its saturated vapor), which is in the state of thermodynamic equilibrium characterized by the given temperature, pressure, and particle number, undergoes irradiation by a source with a constant power. After the time interval of an order of the mean time between the collisions of structural elements (or the characteristic interaction time in liquids), the stochastization of the system takes place, and the distribution function of the particles over their velocities acquires an almost stationary form [19]. The further evolution of the system is exclusively governed by the time dependences of the temperature, pressure, and radiation source power, irrespective of the initial distribution of molecules in the phase space [20,21]. In this case, according to the Prigogine theorem about the stationary state, the system transits into a state with the entropy production minimum [22]. In the range of thermodynamical branch stability, using the hypothesis about the existence of local equilibrium [23], one may unambiguously introduce local thermodynamical functions and consider phase transitions in the framework of equilibrium thermodynamics.

In the framework of this approach, let us consider the influence of irradiation on the evolution of possible phase transitions of the first kind in liquids. The temperature $T_{0}$ and the pressure $p_{0}$ of the corresponding phase transition are determined as solutions of the equations that describe the required conditions of phase equilibrium. In particular, in the case of a one-component liquid, these equations for boiling and crystallization look like [24]:

$\mu_{1}\left(T_{0}, p_{0}\right)=\mu_{2}\left(T_{0}, p_{0}\right)$,

where $\mu_{1}\left(T_{0}, p_{0}\right)$ and $\mu_{2}\left(T_{0}, p_{0}\right)$ are the chemical potentials of a one-component medium in the first and second phases at the phase transition point characterized by the temperature $T_{0}$ and the pressure $p_{0}$ (the basic systems).

Let us consider the case where the influence of a spatially uniform radiation source with a constant power gives rise to the generation of only one kind of new quasiparticles (excited molecules) with the concentration $x_{i}$ in the $i$-th phase of the system. It is important to emphasize that, in the framework of our approach, we do not consider the appearance of charged particles in the system. Then, the dependence of the chemical potential $\mu_{i}\left(T, p, x_{i}\right)$ of nonexcited molecules in this phase on the concentration $x_{i}$ looks like [25]:

$\mu_{i}(T, p)=\mu_{i 0}(T, p)+k T \ln \left[\gamma_{i}\left(T, p, x_{i}\right) x_{i}\right]$,

where $\mu_{i 0}(T, p)$ is the chemical potential of the basic system, and $\gamma_{i}\left(T, p, x_{i}\right)$ the activity coefficient for the subsystem of nonexcited molecules. The relation between the radiation source power and the concentration of excited particles was considered in the literature (see, e.g., works $[17,26]$ ) in detail and is not analyzed in this paper. Note that expression (2) is written for the case where quasiparticles of only one kind are generated under irradiation, but it can be generalized to more complicated cases.

As was mentioned above, the condition of phase equilibrium during the phase transition consists in 
the equality of the chemical potentials of substances in both phases (1). Under the action of radiation, this relation should be rewritten in the following form:

$$
\begin{aligned}
& \mu_{10}\left(T_{\text {ref }}, p\right)+k T \ln \left[\gamma_{1}\left(T_{\text {ref }}, p, x_{1}\right)\left(1-x_{1}\right)\right]= \\
& =\mu_{20}\left(T_{\text {ref }}, p\right)+k T \ln \left[\gamma_{2}\left(T_{\text {ref }}, p, x_{1}\right)\left(1-x_{2}\right)\right]
\end{aligned}
$$

This equality can be satisfied only if the phase transition takes place at a new effective temperature $T_{\text {ref }}$ (in the general case, $T_{\text {ref }} \neq T_{0}$ ), provided a constant pressure. It is easy to see that if excited molecules are available, the curve describing the temperature dependence of the chemical potential (the Gibbs thermodynamic potential) of the liquid becomes shifted by a value that describes the corresponding change of the chemical potential.

Since the concentrations of excited molecules are very low [8], it is evident that the deviation $\delta T$ of the effective phase transition temperature $T_{\text {ref }}$ from the basic one $T_{0}$ is also small $\left(\frac{|\delta T|}{T_{0}} \ll 1\right)$. Therefore, expanding $\mu_{i 0}$ and $\gamma_{i}$ in expression (3) in power series of $\frac{\delta T}{T_{0}}$ up to linear terms, we obtain the following expression for the relative variation of the phase transition temperature:

$$
\begin{aligned}
& \frac{\delta T}{T_{0}}=k T_{0}\left[\ln \frac{1-x_{1}}{1-x_{2}}+\ln \frac{\gamma_{1}}{\gamma_{2}}\right] \times \\
& \times\left[q-k T_{0}\left\{\ln \frac{1-x_{1}}{1-x_{2}}+\ln \frac{\gamma_{1}}{\gamma_{2}}-\right.\right. \\
& \left.\left.-k T_{0}^{2}\left\{\left(\frac{\partial}{\partial T} \ln \gamma_{1}\right)_{p, x_{1}}-\left(\frac{\partial}{\partial T} \ln \gamma_{2}\right)_{p, x_{2}}\right\}\right\}\right]^{-1} .
\end{aligned}
$$

It is worth to note that expression (4) is valid only far from the points of continuous phase transformations $[27,28]$, where the series for the chemical potential converges.

In order to calculate $\frac{\delta T}{T_{0}}$, we have to know the dependence of the activity coefficients $\gamma_{i}$ on the pressure and the concentrations, which are associated with the features in the equation of system state. The explicit form of this dependence is taken rather often from the model of regular solutions or in the form of empirical Margules, Van Laar, or Scatchard-Hammer equations. In this work, we confine the consideration to the cases of a perfect solution, which means the account for only entropic factors, and a regular solution, whose model involves both the entropic and energy contributions.

\section{Ideal Solution}

Let us consider the case where the solution is ideal. In many systems, the solution of excited and unexcited particles with an arbitrary concentration of the former can be considered ideal to a high accuracy, because the components (excited and unexcited molecules) are rather similar to one another with respect to the interaction between the molecules, as well as to their shapes and dimensions [29]. In addition, the solution can often be regarded ideal at $x_{i} \ll 1$. In the framework of this model, the contribution of entropic factors to the thermodynamic potentials at the mixing of unexcited and excited molecules dominates over the energy ones, so that the latter can be neglected. In this case, the activity coefficient is identically equal to unity, and the following expression is obtained for the relative temperature shift $\left(\frac{\delta T}{T_{0}}\right)_{\mathrm{id}}$ :

$\left(\frac{\delta T}{T_{0}}\right)_{\mathrm{id}}=\frac{k T_{0} \ln \frac{1-x_{1}}{1-x_{2}}}{q-k T_{0} \ln \frac{1-x_{1}}{1-x_{2}}}$.

The analysis of this expression demonstrates that, provided the concentration of excited particles is low, the dependence of the temperature shift on $x_{1}$ or $x_{2}$ is linear. At the same time, the sign of $\delta T$ can change if $x_{2}$ is fixed, but the particle concentration in the other phase, $x_{1}$, is varied.

Formula (5) makes it possible to evaluate $\left(\frac{\delta T}{T_{0}}\right)_{\text {id }}$. For instance, for liquid argon coexisting with its saturated vapor at the temperature $T_{0}=87.2 \mathrm{~K}$ and the pressure $p_{0}=1.01 \times 10^{5} \mathrm{~Pa}, \delta T$ can reach a value of $0.12 \mathrm{~K}$ at $x_{2} \approx 0.01$. Such concentrations of excited particles are feasible for micro- and nanosystems [30].

Let us consider the case of the "liquid-vapor" phase equilibrium. The subscript "2" will be used to denote the liquid phase, and subscript " 1 " the vapor one. The concentration of excited molecules depends on the rate of their generation and the rate of their decay, i.e. on their lifetime. The generation rate for excited molecules is proportional to the medium density and the excitation cross-section. In the case of the "liquid-vapor"phase equilibrium, the density of a liquid is larger than that of a vapor. However, the lifetime of vapor molecules in the excited state can be much longer than that of the excited state of molecules in the liquid; for example, if it is determined by the collisions of molecules. Therefore, for a 
one-component medium in a case where the lifetime of the excited state of vapor molecules does not exceed the lifetime of excited state of molecules in the liquid, the relation $x_{2}>x_{1}$ is satisfied, i.e. the boiling temperature increases. Otherwise, the relation $x_{2}<x_{1}$ can be obeyed. In this case, the boiling temperature decreases.

In the case of multicomponent liquid systems, the excitation cross-sections of particles belonging to different components can be different. Therefore, if particles with a larger excitation cross-section are mainly evaporated, the condition $x_{2}<x_{1}$ can be satisfied, and the boiling temperature decreases. Note that the exotic case $T=T_{0}$ may exist, which is realized under the evident condition $x_{2}=x_{1}$.

Now, let us consider the case of the "liquid-solid" phase equilibrium. The subscript " 2 " will correspond to the solid state, and subscript "1" to the liquid one. The densities of the liquid and solid phases of the medium, as well as the excitation cross-sections of molecules in the liquid and solid phases, are almost identical. However, the lifetime of the excited state of molecules in the solid phase, as a rule, is much shorter than that in the liquid, which is related to the fact that, due to the existence of the crystal lattice in a solid, the excitation energy of a molecule can be rapidly redistributed between other molecules of the crystal. As a rule, $x_{2}<x_{1}$, so that the melting temperature decreases. However, if, because of certain reasons, the opposite case takes place, the melting temperature increases. Again, the case $T=T_{0}$ may exist, which is realized provided the condition $x_{2}=x_{1}$.

Finally, let us consider the case of the "gas-solid" phase equilibrium. Now, the subscript "2" will be used to denote the solid and the subscript "1" the gas phase. The excitation cross-sections of molecules in the gas and solid phases of a one-component medium are almost identical. The density of the solid phase considerably exceeds that of the gas phase, but the lifetime of the excited state of molecules in the solid phase is usually considerably shorter than that in the gas one. Therefore, for a one-component medium, various ratios between the numbers of excited molecules in those phases are possible, depending on the specific values of the generation and decay rates for excited molecules in the gas and solid phases. If $x_{2}>x_{1}$, the sublimation temperature increases; otherwise, it decreases.
In the case of multicomponent liquid systems where the excitation cross-sections of particles in different components are different, the ratio between the concentrations of excited molecules in the gas and solid phases can be different for different components. This makes it possible to provide the separation of medium components by means of the medium sublimation under irradiation.

\section{Regular Solution}

Let us consider the case of regular solution. The model of regular solution adequately describes the thermodynamical behavior of electrolyte solutions, but it is also applicable to solid solutions of metals. The activity coefficient of a regular solution can be written in the following form [31]:

$k T \ln \gamma_{i}\left(T, p, x_{i}\right)=\frac{a^{(i)}(p)}{2} x_{i}^{2}$.

Here, with the help of the thermodynamical perturbation theory, the coefficient $a^{(i)}(p)$ can be presented as a combination of integrals $\Phi_{\alpha \beta}^{i}(T, p)$ from the radial distribution function of the basic system, $g_{20}^{i}(\mathbf{r}, T, p)$ [32]:

$$
\begin{aligned}
& \frac{a^{(i)}(p)}{k T}=2 \Phi_{\alpha \beta}^{i}-\Phi_{\alpha \alpha}^{i}-\Phi_{\beta \beta}^{i}, \\
& \Phi_{\alpha \beta}^{i}=\rho_{0}(p, T) \int_{\langle V(p, T, N)\rangle_{0}} d \mathbf{r} g_{20}^{i}(\mathbf{r}, T, p) \times \\
& \times\left[\exp \left(-\frac{\varphi_{\alpha \beta}-\varphi_{0}}{k T}\right)-1\right],
\end{aligned}
$$

where $\rho_{0}(p, T)$ is the numerical density of the basic system, $\varphi_{\alpha \beta}$ the interaction potential between the particles of the $\alpha$-th and $\beta$-th kinds, and $\varphi_{0}$ the interaction potential between particles of the basic system.

In the case of regular solution, on the basis of formula (4), the following expression can be obtained for a relative shift of the phase transition temperature:

$$
\begin{aligned}
& \left(\frac{\delta T}{T_{0}}\right)_{\mathrm{reg}}=\frac{\ln \frac{1-x_{1}}{1-x_{2}}+\frac{a^{(1)} x_{1}^{2}}{2 k T_{0}}-\frac{a^{(2)} x_{2}^{2}}{2 k T_{0}}}{\frac{q}{k T_{0}}-\ln \frac{1-x_{1}}{1-x_{2}}}= \\
& =\left(\frac{\delta T}{T_{0}}\right)_{\mathrm{id}}+\frac{\frac{a^{(1)} x_{1}^{2}}{2}-\frac{a^{(2)}}{2} x_{2}^{2}}{q-k T_{0} \ln \frac{1-x_{1}}{1-x_{2}}} .
\end{aligned}
$$


Hence, this quantity is divided into two terms. The first term is associated only with entropic effects, i.e. with the appearance of new particles in the solution that differ from the basic particles in any, even non-force, characteristics. The second term is connected with a modification of the interaction potential between excited particles. At low concentrations of excited particles, the first term in this parameter is linear, whereas the second is quadratic, which gives rise to the smaller influence of the latter on a shift of the phase transition temperature.

In order to evaluate the contribution of energy factors to a shift of the phase transition temperature according to expressions (7)-(9), we have to know, in addition to the thermal equation of state for the basic system, its radial distribution function $g_{20}^{i}(\mathbf{r}, T, p)$, as well as the potentials of pair interactions "excited particle-excited particle" and "excited particle--unexcited particle". The radial distribution function of argon atoms was found, by using the numerical simulation in the framework of a canonical ensemble and applying the molecular dynamics methods. This procedure was described in work [33] in detail. The molecular dynamics method was realized with the help of the modified software package DL POLY 4.05 [34]. The time step was selected to equal 1 fs. The cubic cell contained 216 interacting particles. Periodic boundary conditions were imposed. The volume of a unit cell in the examined system was calculated in accordance with experimental values obtained for the solution density at the required temperature. The molecule-to-molecule interaction was described using the Lennard-Jones interactomic potential [35].

For illustration, the radial distribution functions of argon atoms at two temperatures are depicted in Figure. The results were obtained with the use of the molecular dynamics methods. They allow us to determine that, in the case of argon at the temperature $T_{0}=87.2 \mathrm{~K}$ and the pressure $p_{0}=1.01 \times 10^{5} \mathrm{~Pa}$, the temperature shift of the "liquid-vapor" phase transition amounts to $(\delta T)_{\text {reg }} \approx 0.11 \mathrm{~K}$ at $x_{1} \approx 10^{-7}$ (gas) and $x_{2} \approx 0.01$ (liquid).

The calculation results obtained for the temperature shift of the first-order phase transition under irradiation testify that, in the framework of the proposed approach, the entropic contributions to this quantity play a dominant role. At the same time, the

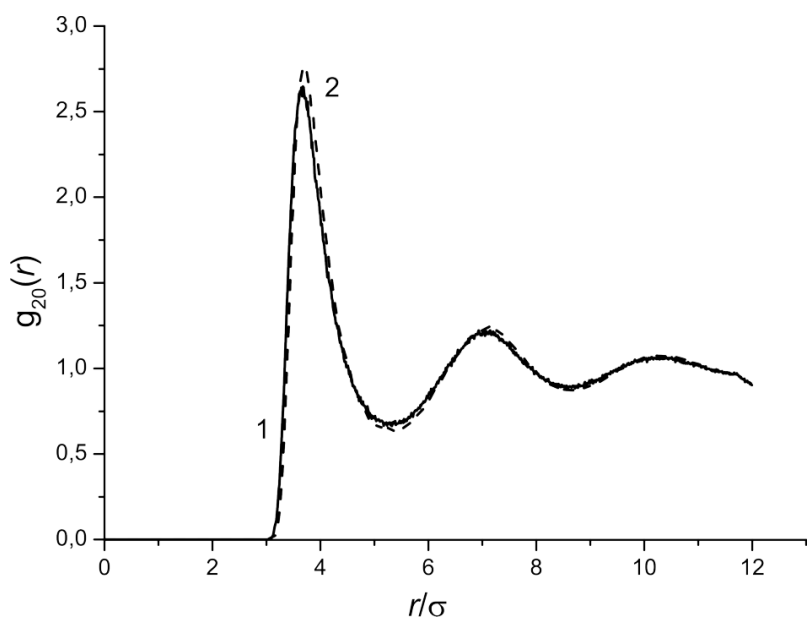

Radial distribution functions for argon atoms at the pressure $p_{0}=1.01 \times 10^{5} \mathrm{~Pa}$ and temperatures of 83 (solid curve 1 ) and $87 \mathrm{~K}$ (dashed curve 2)

account for the energy contributions (the regular solution) gives rise to only an insignificant temperature shift of the phase transition in the system.

\section{Conclusions}

The action of radiation on a liquid system gives rise to an increase of the configurational entropy of the system. As a result, the chemical potentials of the components of a system change, which gives rise, in turn, to the temperature shift of the first-order phase transitions. Depending on the medium properties in various phases (density, scattering and excitation cross-sections, lifetime of excited molecules, and so on) and on the radiation parameters (radiation type, spectrum, flux, and fluence), the phase transition temperature may become shifted at a constant pressure. The entropic contributions to changes of the thermodynamical potentials under the irradiation play a dominant role in the shift of the phase transition temperature in the system. At the same time, the account for the energy contributions (the nonideality of a solution) leads to only an insignificant shift of this temperature. The magnitudes and the signs of corresponding changes are determined by the concentrations of excited molecules in the coexisting phases.

1. I.G. Draganić, Radiolysis of water: a look at its origin and occurrence in the nature, Radiation Phys. Chem. 72, 181 (2005) [DOI: 10.1016/j.radphyschem.2004.09.012]. 
2. T. Pálfi, L. Wojnárovits, and E. Takács, Calculated and measured transient product yields in pulse radiolysis of aqueous solutions: Concentration dependence, Radiation Phys. Chem. 79, 1154 (2010) [DOI: 10.1016/j.radphyschem.2010.06.004].

3. K.O. Trachenko, M.T. Dove, and E.K. Salje, Atomistic modelling of radiation damage in zircon, J. Phys.: Condens. Matter 13, 1947 (2001) [DOI: 10.1088/09538984/13/9/317].

4. K.O. Trachenko, M.T. Dove, T. Geisler, I. Todorov, and B. Smith, Radiation damage effects and percolation theory, J. Phys.: Condens. Matter 16, S2623 (2004) [DOI: 10.1088/0953-8984/16/27/002].

5. K. Trachenko, E. Zarkadoula, I. Todorov, M. Dove, D. Dunstan, and K. Nordlund, Modeling high-energy radiation damage in nuclear and fusion applications, Nucl. Instr. Meth. Phys. Rev. B 277, 6 (2012) [DOI: 10.1016/ j.nimb.2011.12.058]

6. E. Zarkadoula et al., The nature of high-energy radiation damage in iron, J. Phys.: Condens. Matter 25, 125402 (2013) [DOI 10.1088/0953-8984/25/12/125402].

7. L. Malerba et al., Ab initio calculations and interatomic potentials for iron and iron alloys: Achievements within the Perfect Project, J. Nucl. Mater. 406, 7 (2010) [DOI: 10.1016/j.jnucmat.2010.05.016].

8. I.A. Shkrob, T.W. Marin, S.D. Chemerisov, and J.F. Wishar, Radiation induced redox reactions and fragmentation of constituent ions in ionic liquids. 1. Anions, J. Phys. Chem. B 115, 3872 (2011) [DOI: 10.1021/jp2003062].

9. K. Trachenko et al., Radiation damage in the bulk and at the surface, Mol. Simulat. 31, 355 (2005) [DOI: 10.1080/08927020500066825].

10. V.V. Brazhkin et al., "Liquid-gas" transition in the supercritical region: Fundamental changes in the particle dynamics, Phys. Rev. Lett. 111(14), 145901 (2011) [DOI: 10.1103/PhysRevLett.111.145901].

11. A.V. Chalyi, L.A. Bulavin, V.F. Chekhun et al., Universality classes and critical phenomena in confined liquid systems, Condens. Matter Phys. 16, 23008 (2013) [DOI: 10.5488/CMP.16.23008].

12. A.V. Chalyi and E.V. Zaitseva, A kinetic model of synaptic transmission on intercell interaction, Ukr. J. Phys. 54, 366 (2009).

13. A.V. Chalyi and E.V. Zaitseva, Strange attractor in kinetic model of synaptic transmission, J. Phys. Studies 11, 322 (2007).

14. V.M. Novikov, V.V. Ignat'ev, V.I. Fedulov, and V.N. Cherednikov, Liquid Salt NEI: Perspectives and Problems (Energoatomizdat, Moscow, 1990) (in Russian) [ISBN: 5-28303791-6].

15. M. Rosenthal, R. Briggs, and P. Haubenreich, Molten-salt reactor program. Semiannual progress report for period ending August 31, 1971, preprint ORNL-4728 (1972).
16. S.A. Bznuni, V.S. Barashenkov, and V.M. Zhamkochyan, Perspective two-reactor electronuclear systems, Fiz. Elem. Chast. At. Yadra 34977 (2003).

17. A.K. Pikaev and V.I. Spitsyn, Modern Radiation Chemistry: Radiolysis of Gases and Fluids (Nauka, Moscow, 1986) (in Russian).

18. D.A. Gavryushenko, The influence of irradiation on phase transition properies i n fluid systems, Dopov. Nats. Akad. Nauk Ukr., No. 8, 83 (2013).

19. D.N. Zubarev, Mechanics (Consultants Bureau, New York, 1974).

20. M. Kac, Probability and Related Topics in Physical Sciences (Amer. Math. Soc., New York, 1959).

21. I. Prigogine, Mechanics (Interscience, New York, 1962).

22. I. Prigogine, Introduction to Thermodynamics of Irreversible Processes (Interscience, New York, 1962).

23. S.R. de Groot and P. Mazur, Nonequilibrium Thermodynamics (North-Holland, Amsterdam, 1963).

24. L.A. Bulavin, D.A. Gavryushenko, and V.M. Sysoev, Molecular Physics (Znannya, Kyiv, 2006) (in Ukrainian).

25. D. Kondepudi and I. Prigogine, Modern Thermodynamics: from Heat Engines to Dissipative Structures (Wiley, New York, 2014).

26. P.A. Selishchev, Self Organization in Radiation Physics (Aspekt-Poligraf, Kiev, 2004) (in Russian).

27. M.P. Kozlovskii and R.V. Romanik, Influence of an external field on the critical behavior of the 3D Isinglike model, J. Molec. Liq. 167, 14 (2012) [DOI: 10.1016/j.molliq.2011.12.003].

28. M.P. Kozlovskii, Recurrence relations for the threedimensional Ising-like model in the external field, Condens. Matter. Phys. 8, 473 (2005).

29. K. Trachenko, J.M. Pruneda, and E. Artacho, How the nature of the chemical bond governs resistance to amorphization by radiation damage, Phys. Rev. B 71, 184104 (2005) [DOI: 10.1103/PhysRevB.71.184104].

30. M. Chiapetto, C.S. Becquart, C. Domain, and L. Malerba, Nanostructure evolution under irradiation of $\mathrm{Fe}(\mathrm{C}) \mathrm{MnNi}$ model alloys for reactor pressure vessel steels, Nucl. Instr. Methods Phys. Res. B 352, 56 (2015) [DOI: 10.1016/j.nimb.2014.11.102].

31. V.A. Durov and E.P. Ageev, Thermodynamical Theory of Solutions of Nonelectrolytes (Moscow State Univ., Moscow, 1987) (in Russian).

32. V.M. Sysoev and S.A. Terletskii, On the influence of the third component on the mutual solvability of two fluids, Zh. Fiz. Khim. 58, 370 (1984).

33. N.A. Atamas, L.A. Bulavin, V.I. Kovalchuk, and A.M. Mayko, Influence of radiation on the local structure in a $\mathrm{NaCl}$ aqueous solution, Ukr. J. Phys. 60, 422 (2015).

34. T. Schlick, Molecular Modeling and Simulation: An Interdisciplinary Guide (Springer, New York, 2002).

35. M.P. Allen and D.Y. Tildesley, Computer Simulation of Liquids (Clarendon Press, Oxford, 2010).

Received 31.01.2016. Translated from Ukrainian by O.I. Voitenko ISSN 2071-0194. Ukr. J. Phys. 2016. Vol. 61, No. 9 
Л.А. Булавін, Д.А. Гаврюшенко,

K.В. Тарадій, Н.О. Атамасъ, В.М. Сисоєв

ВПЛИВ РАДІАЦІЙНОГО

ОПРОМІНЕННЯ НА ТЕМПЕРАТУРУ

ФАЗОВИХ ПЕРЕХОДІВ В РІДИНАХ

$\mathrm{P}$ е 3 ю м е

Досліджено вплив радіаційного опромінення на термодинамічні властивості рідинних систем, які визначаються змі- ною хімічного потенціалу рідини та її компонентів під дією випромінювання. Показано, що радіаційне опромінення співіснуючих фаз в стаціонарному стані приводить до зсуву параметрів точок фазових переходів. Визначено зміну температури фазових переходів першого роду під дією радіаційного опромінення шляхом врахування як ентропійного, так і енергетичного фактора в хімічному потенціалі системи. 